\title{
PAUTAS PARA LA INTEGRACION DE LOS ESTUDIANTES UNIVERSITARIOS CON DISCAPACIDAD AUDITIVA Y VISUAL ANTE EL DESAFIO DEL EEES
}

María Yolanda Martínez-Solana1: Universidad Complutense de Madrid. España mymartinez@ccinf.ucm.es

\section{RESUMEN}

El modelo de enseñanza universitaria emanado del Plan Bolonia es más avanzado que los previamente existentes ya el que el alumno pasa de la docencia por enseñanza, basada en la recepción de conocimientos, a la docencia basada en el aprendizaje, planteada sobre desarrollo de competencias en los estudiantes. Se necesita una formación más ambiciosa del profesorado que enseñe a aprender y, por tanto, exige de los estudiantes una mayor responsabilidad en su propia educación, algo que no hace sino agravar las dificultades de los alumnos con deficiencias sensoriales. Siguen existiendo barreras en el ámbito universitario de cara al proceso de aprendizaje de las personas que integran este colectivo, originadas sobre todo, por una todavía deficiente organización educativa y un casi inexistente material académico adaptado a las necesidades especiales que requieren las personas con diferentes tipos de discapacidad. Con la entrada en vigor del llamado Plan Bolonia y de unos currículums en los que el alumno cada vez es más protagonista de su propio aprendizaje, estas barreras son aún más acusadas.

PALABRAS CLAVE: Universidad - Discapacidad - Adaptación - Currículo formativo - Materiales docentes

\footnotetext{
${ }^{1}$ Autor Correspondiente

Profesora Contratado Doctor. Facultad de Ciencias de la Información. Universidad Complutense de Madrid. España.

Correo: $\underline{\text { mymartinez@ccinf.ucm.es }}$
} 


\title{
GUIDELINES FOR THE INTEGRATION OF UNIVERSITY STUDENTS WITH VISUAL AND HEARING DISABILITIES TO THE CHALLENGE OF EEES
}

\begin{abstract}
The called Bologna Process is a more advanced model in which the student changes teaching and learning based in knowledge reception, to a new education, raised on skills development in students. This system demand training teachers to teach to learn and, therefore, it requires a bigger responsibility of the students in their own education, something that does nothing but aggravate the difficulties of disabled students. We can see as barriers still exist in the university setting towards the learning process of individuals making up this group originated mainly by a poor yet educational organization and an almost non-existent academic material adapted to the special needs that require people with different types of disabilities. With the entry into force of the so called Bologna Process and a curriculum in which the student is becoming protagonists of their own learning these barriers are even more significant.
\end{abstract}

KEY WORDS: University - Disability- Adaptation - training curriculum - teaching materials

\section{INTRODUCCIÓN}

Los estudiantes con discapacidad visual o auditiva que acceden a la universidad aún se encuentran barreras para un correcto seguimiento de las actividades formativas. Esencialmente esa realidad es el resultado de una todavía deficiente organización educativa y un casi inexistente material académico adaptado a las necesidades especiales que requieren las personas con diferentes tipos de discapacidad. Con la entrada en vigor del llamado Plan Bolonia y de unos currículums en los que el alumno cada vez es más protagonista de su propio aprendizaje, estas barreras son aún más acusadas.

La existencia de una discapacidad no debería conllevar una merma adicional en el proceso de aprendizaje. Todos los estudiantes han de poder participar con igualdad de oportunidades. Por lo tanto, se tienen que eliminar todas aquellas barreras que lo impidan. 
Las universidades públicas en nuestro país tienen matriculados 7.408 alumnos con discapacidad, el $0.53 \%$ del total de alumnado, según datos del Libro Blanco sobre Discapacidad en la Universidad realizado en base a datos del CERMI. En cuanto a las universidades privadas, y con los datos disponibles, el porcentaje de alumnado con discapacidad es del $0.24 \%$. La LOU exige la atención individualizada a los estudiantes con discapacidad, que se extiende a toda su estancia en la universidad e incluye la correspondiente adaptación curricular.

Las personas con necesidades educativas específicas han de poder integrarse en los grupos de alumnos y seguir la docencia impartida sin necesidad de recurrir a medidas excepcionales. La adaptación de contenidos de las asignaturas de Periodismo, Publicidad y Relaciones Públicas, Comunicación Audiovisual y Ciencias Físicas y la puesta a disposición de los alumnos con discapacidad de prácticas para el desarrollo de los conocimientos de las materias en formatos accesibles y fácilmente manejables para ellos ha sido el objetivo central de la autora, también con el propósito de lograr la meta de dotar al profesorado de instrumentos para el desarrollo de su labor docente con una metodología basada en las últimas tecnologías que permita su formación continuada y que le liberase de la necesidad de buscaradaptaciones curriculares improvisadas para los estudiantes con discapacidad.

La finalidad del proceso de adaptación aplicado cubría un doble objetivo, mejorar la atención a los estudiantes, concretamente a un colectivo como el de los que sufren algún tipo de discapacidad, y, a la vez, incidir en la formación del profesorado de cara a que pueda cumplir, con mejores medios, el reto de mejorar la docencia universitaria hasta situarla en el nivel de excelencia que la lleve a liderar el Espacio Europeo de Educación Superior.

Se trataba de enriquecer con la innovación tanto las clases teóricas, como las prácticas, los seminarios y las tutorías de todos los estudiantes que conviven cada día con algún tipo de discapacidad es un reto apasionante y, para ello, entendemos que es necesario diseñar metodologías activas y participativas orientadas a facilitar su completa integración en las aulas. Por desgracia, actualmente las políticas de adaptación curricular de este tipo de alumnos se ejecutan, muchas veces, con discrecionalidad o como medidas de gracia del profesor, a falta de una normativa que las concrete y regule $\mathrm{y}$, quizás lo más importante, de una normativa que comprometa a todas las universidades en una misma dirección y las dote de recursos y ayudas técnicas.

A pesar que ha de reconocerse que las políticas de apoyo a la discapacidad han evolucionado de forma considerable en los últimos años. Normalmente, el aula no 
Y no lo está, entre otras cuestiones, por la dificultad que entrañan las políticas generalistas.

Una característica importante de esta población estudiantil específica, a la que este proyecto de innovación pretende ofrecer soluciones concretas, es la diversidad. Nos hallamos ante un grupo de personas muy diversas y con necesidades especificas muy diferentes. Por ello, la inclusión de los estudiantes con discapacidad a los estudios superiores requiere un análisis minucioso de las necesidades de este colectivo. Asimismo se estima que el $64 \%$ de los profesores universitarios ha tenido alguna vez a algún alumno con discapacidad, lo que demuestra que este proyecto no sólo beneficiará a un importantísimo número de estudiantes sino que puede ser de utilidad al grueso de cuerpo de profesores que reconoce sentirse "incómodo" cuando esto sucede ante la constante sensación de que no responde satisfactoriamente a las necesidades específicas de este tipo de estudiantes.

\section{METODOLOGÍA}

La inclusión en el equipo de dos estudiantes con discapacidad visual ha sido el eje esencial de la metodología empleada, que ha estado abierta a cualquier sugerencia práctica percibida por los estudiantes -usuarios conforme íbamos avanzando los trabajos. Para ello, el equipo ha mantenido reuniones quincenales en las que por un lado los profesores recibíamos las necesidades puntuales de los alumnos invidentes $\mathrm{y}$, por otro, planteábamos las posibles soluciones. Con el fin de solventar los problemas que cada día tienen que padecer los estudiantes discapacitados procedimos a dar, en cada uno de los libros y apuntes de las asignaturas tratadas, los siguientes pasos:

a) Realización del escaneo y reconocimiento de cada uno de los textos para que estén disponibles en formato Word.

b) Cada una de las páginas de dichos documentos se han convertido a formato MP3 con la finalidad de que los estudiantes cuenten con cada libro en formato audio para almacenarlo en su reproductor de MP3 para tener un medio alternativo de estudio y no depender del todo de un ordenador.

c) Debido a la dificultad que presentan los textos en archivo informático para poder desplazarse por las páginas para un estudiante ciego, realizamos una guía en formato braille para cada una de las asignaturas donde se describen los nombres de capítulos, temas, subtemas, apartados, indicando su localización en cada página y de esta manera haciendo posible encontrar de manera más rápida los puntos ya señalados. La gran cantidad de material de lectura que exige una carrera universitaria hace que el sistema de 


\section{ANÁLISIS Y DISCUSIÓN}

La transformación en realidad del proyecto ha sido una tarea gratificante, tanto por la interrelación con los estudiantes que han participado en él, como por los resultados obtenidos. Creemos que con ellos se ha abierto una línea de investigación interdisciplinar e innovadora que adecua todos los contenidos necesarios para el seguimiento de tres asignaturas de las Licenciaturas de Publicidad y Relaciones Públicas y Periodismo a las necesidades de los alumnos con discapacidad visual y auditiva. Al final del proyecto hemos conseguido acabar con una de las barreras académicas más significativas con las que se encuentran los estudiantes con discapacidad visual, la utilización de materiales bibliográficos. El estudiante con discapacidad visual, a diferencia de otro estudiante sin esta minusvalía, debe realizar los siguientes pasos para acceder a la información:

a) conseguir el material bibliográfico de manera impresa.

b) realizar el proceso de reconocimiento de textos a través de medios informáticos, (escáner, ordenador y programas especializados).

c) tras el escaneo, se debe realizar la revisión y proceso de lectura por parte del procesador de textos instalado en el ordenador.

d) leer y realizar la extracción de los datos del interés del estudiante.

Un estudiante sin discapacidad visual solo debe adquirir el libro, texto o apuntes correspondientes y sentarse a leer, por lo que el proceso antes descrito significa una inversión de tiempo fundamental para el estudiante ciego y por ende, contar con menos tiempo destinado al estudio concreto de la información que debe extraer del texto a leer.

Cada uno de los libros se encuentra disponible y accesible para estudiantes con discapacidad audiovisual. De esta manera hemos hecho realidad el objetivo inicial que nos movió de adaptar los contenidos de distintas asignaturas a formato mp3, con el fin de facilitar su accesibilidad a todo el colectivo de discapacitados y en particular a los que sufren problemas auditivos y visuales. Hemos conseguido adaptar los mismos contenidos, prácticas y herramientas de trabajo del resto de sus compañeros, no hemos creado unos específicos ni, por supuesto, unos de menor nivel para ellos. Pero a medida que íbamos avanzando en esta tarea hemos descubierto la existencia de algunas inequidades a la hora de que los estudiantes con alguna discapacidad puedan equipararse realmente al resto de compañeros en el desarrollo, sobre todo, de las prácticas que la adaptación de las titulaciones al Espacio Europeo de Educación 
Tal es el caso de la posibilidad de llevar a cabo presentaciones o exposiciones que precisen del uso de la mayoría de los programas informáticos (excepto Word) que los estudiantes utilizan de manera habitual, tales como Excel o Power Point. Hasta el momento es inexistente un manual de utilización de programas básicos pero fundamentales en diversas tareas de tipo académico como las hojas de cálculo (Excel) y la elaboración de presentaciones (Power Point).

Si realmente deseamos una apertura total a la educación, una formación profesional sin barreras de ningún tipo, entonces debemos pensar que un estudiante con discapacidad no puede depender solamente del procesador de textos y de acuerdo a las exigencias de su carrera deberá contar con un manejo de otras herramientas de tipo ofimático que le permitan realizar sus actividades con total independencia y conocimiento de la organización de las mismas.

Asimismo, hemos podido comprobar que existe otro campo en el que los estudiantes videntes y los que no lo son compiten de forma totalmente desigual. Toda bibliografía escrita en inglés es un obstáculo insalvable para los estudiantes discapacitados, dada la carencia absoluta de cursos de inglés técnico y especializado adaptados para ellos. Se hace necesaria y urgente la elaboración de materiales bibliográficos como diccionarios temáticos, etimológicos y ejercicios prácticos con ejemplos en inglés.

El Proyecto se abordo en dos vertientes, la primera, dentro del aula, en la que se ha impulsado el diseño de los programas de las asignaturas y las metodologías utilizadas cuando se imparten. La segunda ha estado enfocada a la producción de materiales y herramientas docentes accesibles para los estudiantes con discapacidad, con el fin de que éstas tendiesen a contemplar la diversidad y por tanto se aproximasen cada vez más a una metodología inclusiva que sin duda creemos que ha favorecido y favorecerá que las personas con discapacidad puedan llevar a término su proyecto formativo con las mismas oportunidades que el resto de estudiantes universitarios.

Se han adaptado a formato MP3 los libros de "La Comunicación Institucional: Análisis de sus problemas y soluciones", de Yolanda Martínez Solana; "Teoría de La Comunicación Audiovisual", y los apuntes de la asignatura "Periodismo de Investigación". Para todas estas asignaturas se ha editado en braille una guía mediante la cual los alumnos invidentes pueden seguir página a página el contenido del MP3 sin necesidad de disponer de un ordenador.

Este sistema dota de gran agilizad al sistema de búsqueda de capítulos y apartados 
Las páginas que se mencionan son las páginas del libro original, no las que indica Word, ya que cuando en clase el profesor solicita o señala algún concepto suele guiarse por el libro físico y no por el Word de que dispone el invidente. Como ejemplo adjuntamos una pequeña parte de la guía desarrollada para un capítulo.

Basta indicar que la página 19 del libro es la página 1 en el archivo de Word, por lo que existe una diferencia de 19 páginas entre sí, lo que hasta ahora dificultaba muchísimo a los invidentes seguir correctamente las clases.

Creemos que el material diseñado facilita el acceso al autoaprendizaje por parte del alumno discapacitado y un mayor desarrollo de sus capacidades y autonomía personal. Entendemos que se hacía necesario, además de la traducción de los contenidos académicos al sistema braille, audiodescribirlos también, sobre todo en aquellos casos en el que el contenido docente se hacía más didáctico, explicativo y transparente mediante su representación a través de gráficos, esquemas $u$ otros métodos didácticos de compleja transcripción al braille.

\subsection{Propuestas para el cambio de modelo}

Entre las conclusiones extraídas tras la experiencia realizada destaca la necesidad de dotar a los alumnos con discapacidad de personal de apoyo que sirva de intermediario entre el alumno y el ordenador (por ejemplo mediante las tradicionales becas de colaboración) a fin de facilitar el acceso a estos importantes sistemas que facilitan el autoaprendizaje del alumno.

La dirección de este Proyecto, y su posterior materialización permite concluir que es imprescindible realizar una variación en el modelo de currículo adaptado a las personas con necesidades educativas específicas, facilitando el aprendizaje de todos los alumnos en su diversidad. De esta forma se podrá admitir la posibilidad de acceder a los mismos recursos disponibles para todos en la Universidad desde horizontes particulares y distintos, pero no por ellos menos legítimos.

\section{CONCLUSIONES}

Como una forma de acercamiento a la realidad de la gestión de las necesidades de alumnos con discapacidad hemos establecido unas ideas clave, plasmadas en las siguientes conclusiones:

a) La adaptación curricular para estudiantes con discapacidad visual o auditiva no debe ser una medida discrecional del profesor. Se trata de reajustar todos y 
uno de los alumnos/as.

b) Los créditos ECTS expresan un auténtico cambio metodológico en la Educación Superior Europea. Un modelo más avanzado en el que el alumno pasa de la docencia por enseñanza, basada en la recepción de conocimientos, a la docencia por aprendizaje, planteada sobre desarrollo de competencias. Este sistema demanda del profesorado una formación que enseñe a aprender, y exige de los estudiantes una mayor responsabilidad en su propia educación, algo que no hace sino agravar las dificultades de los alumnos con deficiencias sensoriales.

c) Los nuevos créditos europeos valoran el tiempo invertido por el alumno para adquirir las competencias del programa de estudios -cada uno representa entre 25 y 30 horas de aprendizaje- lo que incluye no sólo la asistencia al aula, sino también la dedicación al estudio, la realización de seminarios, resolución de ejercicios, etc. En el caso de los alumnos con discapacidad hay que pensar, de antemano, cómo se valorará el tiempo extra de trabajo que deben llevar a cabo para cada actividad. Hay estudios que estiman que en un examen normal los alumnos invidentes necesitan al menos entre un 25 y un $50 \%$ más de tiempo para que el resto de los estudiantes, por lo que éste puede ser un dato aproximado para valorar los tiempos en el resto de las actividades.

d) La gran cantidad de material de lectura que exige una carrera universitaria hace que el sistema de impresiones braille se vuelva poco práctico. Por lo tanto, los estudiantes con discapacidad visual deberían poder disponer de bibliografía de las asignaturas y apuntes de clase en formatos accesibles, principalmente textos electrónicos en MP3.

e) Sin perjuicio de que, en virtud del principio de equidad, el nivel de exigencia sea el mismo para todos los alumnos y alumnas, hay que ajustar la evaluación al proceso de aprendizaje efectivamente realizado por los alumnos con discapacidad. En el caso de que se hayan realizado adaptaciones curriculares de algún objetivo y/o contenido, éstas tendrán que ser tenidas en cuenta a la hora de diseñar las pruebas. Éstas versarán sobre las capacidades alternativas que, de hecho, se han desarrollado

f) Una de las claves del tema de la accesibilidad de los materiales para invidentes y discapacitados auditivos es el elevado coste económico de los productos. La tecnología debe estar al alcance de todos, sobre todo de las personas con discapacidad por lo que significa para ellas en calidad de vida. Se trata de trabajar para que cada persona tenga derecho al acceso a la tecnología a través de una sociedad con conciencia social que así lo permita.

g) Los programas de integración deberían trabajar en red entre ellos, especialmente en el contexto europeo, promoviendo el intercambio de experiencias de buena práctica. La creación de listas de distribución y 
h) Hay que incrementar la participación de los alumnos con discapacidad en la gestión de su asignatura. No deben ser contemplados como sujetos pasivos sino como protagonistas de su propio proyecto educativo y programa de integración y, también de su evaluación.

\section{Legislación y marco legal}

- Ley Orgánica 6/2001, de 21 de diciembre, de Universidades. De la integración de los estudiantes con discapacidad en las universidades.

- R.D. 696/1995 de 28 de Abril, de ordenación de los alumnos con NEE Art.6.3

\section{REFERENCIAS}

Parrilla, A. \& MORIÑA, A. (2004). Lo que todos nos preguntamos sobre la educación inclusiva. Padres y Maestros, 284: 10-14.

Shepard, Lorrie A. (2000a). The Role of Classroom Assessment in Teaching and Learning. Los Angeles: CRESST - CSE Technical Report 517.

Tolrà Mabilon, J. (1997). PIUNE. Programa de integración de los universitarios con necesidades especiales. En Universidad y Discapacidad. Valencia: Lliso.

\section{María Yolanda Martínez Solana}

Doctora en Periodismo y Profesora de la Facultad de CC. de la Información de la Universidad Complutense de Madrid. Profesora Contratado Doctor. Miembro de la Sociedad Española de Periodística. Miembro de la Federación de Asociaciones de la Prensa de España. Jurado de los Premios de Periodismo y Responsabilidad Social Empresarial (FORÉTICA). Experta en Información sobre Ciencias de la Salud y aplicación de la comunicación en instituciones públicas y privadas. Autora de varios libros y artículos científicos en relación con estos temas. Directora de Practicum del Máster del Máster Universitario en Investigación en Periodismo: discurso y comunicación de la UCM, así como de Tesis Doctorales. 